\title{
THE PATTERN ELECTRORETINOGRAM IN ANTERIOR VISUAL PATHWAY DYSFUNCTION AND ITS RELATIONSHIP TO THE PATTERN VISUAL EVOKED POTENTIAL: A PERSONAL CLINICAL REVIEW OF 743 EYES
}

\author{
GRAHAM E. HOLDER \\ London
}

\begin{abstract}
SUMMARY
The pattern electroretinogram (PERG) has now been in routine clinical use for sufficiently long to allow a personal clinical review of its relationship to the cortically generated pattern visual evoked potential (PVEP). The PERG and PVEP findings are presented from 520 eyes with optic nerve demyelination (382 eyes), optic nerve compression (90 eyes) or heredofamilial optic atrophy (48 eyes), and these are compared with the findings obtained in 223 eyes with dysfunction anterior to the retinal ganglion cells. Dysfunction anterior to the retinal ganglion cells gives a reduction in the P50 component of the PERG, but this component is usually spared in optic nerve disease where selective loss of the $\mathbf{N} 95$ component is by far the most frequently occurring abnormality. A diagnostic strategy is presented.
\end{abstract}

Although Riggs and co-workers ${ }^{1}$ first reported that small retinal potentials are evoked when a pattern is reversed in contrast, the development of the Arden gold foil corneal electrode, ${ }^{2}$ synchronous with the introduction of the DTL thread electrode ${ }^{3}$ facilitated the introduction of the pattern electroretinogram (PERG) into routine clinical practice. This paper offers a personal clinical review of the PERG, as this test has now been in routine use in the author's laboratory for more than 10 years, and has become an invaluable part of the diagnostic armamentarium. The review examines the use of the PERG in conjunction with the pattern visual evoked potential

From: Regional Neurosciences Unit, Department of Clinical Neurophysiology, King's College Hospital, London; and Electrodiagnostic Department, Moorfields Eye Hospital, London, UK.

Correspondence to: Dr Graham E. Holder, Electrodiagnostic Department, Moorfields Eye Hospital, City Road, London EC1V 2PD, UK. Tel: +44-171-566-2120. Fax: +44-171-566-2465.
(PVEP) in the assessment of macular and optic nerve function. After a summary and review of the PERG and PVEP results in various conditions, a diagnostic strategy is presented.

There are two main approaches for recording a PERG: transient and steady state. The transient PERG is a discrete response obtained with a stimulus rate of less than 7 reversals per second. The main components of the transient PERG are a positive component, P50, at approximately $50 \mathrm{~ms}$ and a larger negative component, N95, at some $95 \mathrm{~ms}^{4,5}$ Many subjects have a small early negativity (N35) at 30-35 ms. A steady-state response can be obtained by using a faster reversal rate. An $8 \mathrm{~Hz}$ stimulus rate (i.e. 16 reversals per second) is commonly used. The measurement of individual components is not possible with steady-state recording, and although it may be a useful research tool, this limits its routine clinical use; the steady-state PERG will not be further discussed in detail.

Both the PERG P50 component and the b-wave of the ERG are positive components, and it is perhaps this superficial waveform similarity that led many early workers on the origins of the PERG, and much early clinical work, to examine this component in isolation. There were no conclusive data (for review see Berninger and $\operatorname{Arden}^{6}$ ). Some animal work implicated the ganglion cells, ${ }^{7,8}$ but the failure of some authors ${ }^{9,10}$ to observe low spatial frequency attenuation of the P50 component of the PERG in man was against this. Indeed, at one stage Spekreijse's group proposed that the PERG may simply be a summation of local luminance changes. ${ }^{11}$

Our perceptions changed when attention was focused on both the P50 and N95 components of the PERG. Berninger and Schuurmans ${ }^{12,13}$ found 
that although the P50 component failed to show spatial tuning, this was present for the N95 component, suggesting a ganglion cell site of origin for N95, but not necessarily for P50. They suggested that N95 was a contrast-dependent component, but that there was a significant luminance contribution to P50. Spekreijse's group later showed that at least $50 \%$ of the PERG is contrast related for check sizes subtending less than 120 ' of arc. ${ }^{14}$ Drasdo's group, with Arden, ${ }^{15}$ extracted the 'pattern specific response' (PSR) ${ }^{16}$ from the PERG and demonstrated a correlation between the amplitude of the negative PSR (N95 equivalent) and the volume of the ganglion cell layer, providing further experimental evidence for a ganglion cell origin for N95.

Over the same period, the clinical observations that P50 and N95 may be selectively affected in disease processes, ${ }^{47-19}$ provided clinical evidence in keeping with Berninger and Schuurmans' suggestion that the two main components may have different origins. Following this, Celesia's group ${ }^{20}$ using a cat model, demonstrated that the effect of optic nerve section was more marked on the late negative (N95 equivalent) than the positive component; there are, however, problems when extrapolating from cat retina to human. Overall, it seems probable that N95 and some of P50 are ganglion cell derived, but that there may be a contribution to the P50 component from more distal retina.

\section{TECHNICAL CONSIDERATIONS AND METHODOLOGY}

The development of the Arden gold foil electrode ${ }^{2}$ and the DTL fibre electrode ${ }^{3}$ made PERG recording a clinically feasible proposition. Both are relatively easy to apply with minimal risk of trauma to the cornea and leave the optics of the eye unimpaired essential when viewing a structured stimulus. Topical anaesthesia is not usually required, and is probably contraindicated. ${ }^{21}$ The practical details of gold foil ${ }^{22}$ and DTL ${ }^{23}$ electrode application are described elsewhere. The positional stability of the gold foil electrode (when correctly applied) is probably greater than that of the DTL, but requires greater experience to attain competence in application. Surface electrodes cannot be recommended for routine use due to the marked and variable degree of amplitude attenuation, but may have a role in paediatric practice. Although there have been claims that the trial-to-trial variability of the PERG is too great for it to be a clinically useful tool, ${ }^{24}$ or that the gold foil is an unsatisfactory electrode, ${ }^{25}$ these have not been confirmed by studies that stringently controlled for technical variables, ${ }^{21,26}$ and the importance of technique and technical factors in the recording of clinical PERGs cannot be over-emphasised. Of particular importance are the use of an outer canthus reference electrode to avoid contamination from the cortically generated PVEP; the setting of an appropriate artefact rejection level during signal averaging; the use of amplifiers that do not saturate when a high-amplitude artefact occurs (such as a blink); and the correct positioning of both electrode and patient.

All PERG data presented in this review were obtained using Arden gold foil electrodes in relation to ipsilateral outer canthus reference electrodes (silver/silver chloride). Bandpass was $1-100 \mathrm{~Hz}$, artefact reject was $60 \mu \mathrm{V}$ peak-to-peak, analysis time was $180 \mathrm{~ms}$, there were a minimum of 150 averages per trial, often more, and a minimum of two trials. The stimulus was a black and white moving mirror checkerboard subtending a total field of $14^{\circ}$ with an individual check size of 34 minutes of arc. There was a central luminance of $400 \mathrm{~cd} / \mathrm{m}^{2}$, dropping off to $200 \mathrm{~cd} / \mathrm{m}^{2}$ at the corners. All recordings were taken binocularly using the 'interrupted stimulation' technique previously described ${ }^{26}$ and a stimulus rate of 4.8 reversals/s $(2.4 \mathrm{~Hz})$. The appropriate refraction was used.

All PVEP data were obtained using the same stimulator, but with 26 minutes of arc checks in a $13^{\circ}$ total field. Electrodes were placed according to the Modified Maudsley system of electrode placement as previously described. ${ }^{27}$ Bandpass was $1-100 \mathrm{~Hz}$, artefact reject was $100 \mu \mathrm{V}$ peak-to-peak, analysis time was $400 \mathrm{~ms}$, there were a minimum of 100 averages per trial, often more, and a minimum of two trials. All recordings were taken monocularly using a stimulus rate of 1.95 reversals/s. The appropriate refraction was used.

\section{RESULTS AND DISCUSSION}

Given the nature of this paper, an approach to the data that combines Results and Discussion for the different disease categories has been adopted.

\section{Retinal and Macular Disease}

The PERG findings from a large group of patients (223 eyes) with generalised retinal or macular disease are shown in Figs. 1 and 2 in relation to the latency of the P100 component of the PVEP. They are patients either with dysfunction confined to the macula (e.g. macular dystrophy, central serous retinopathy), or with macular involvement as part of generalised retinal dysfunction (e.g. retinal dystrophy, inflammatory retinopathy). The data in these, and subsequent figures, are presented as both the absolute values of the P50 component amplitude, and also the ratio between the N95 component amplitude and the P50 component amplitude. The N95:P50 ratio remains normal if a reduction in P50 is reflected in N95, but will be reduced if there is selective loss of the N95 component. 


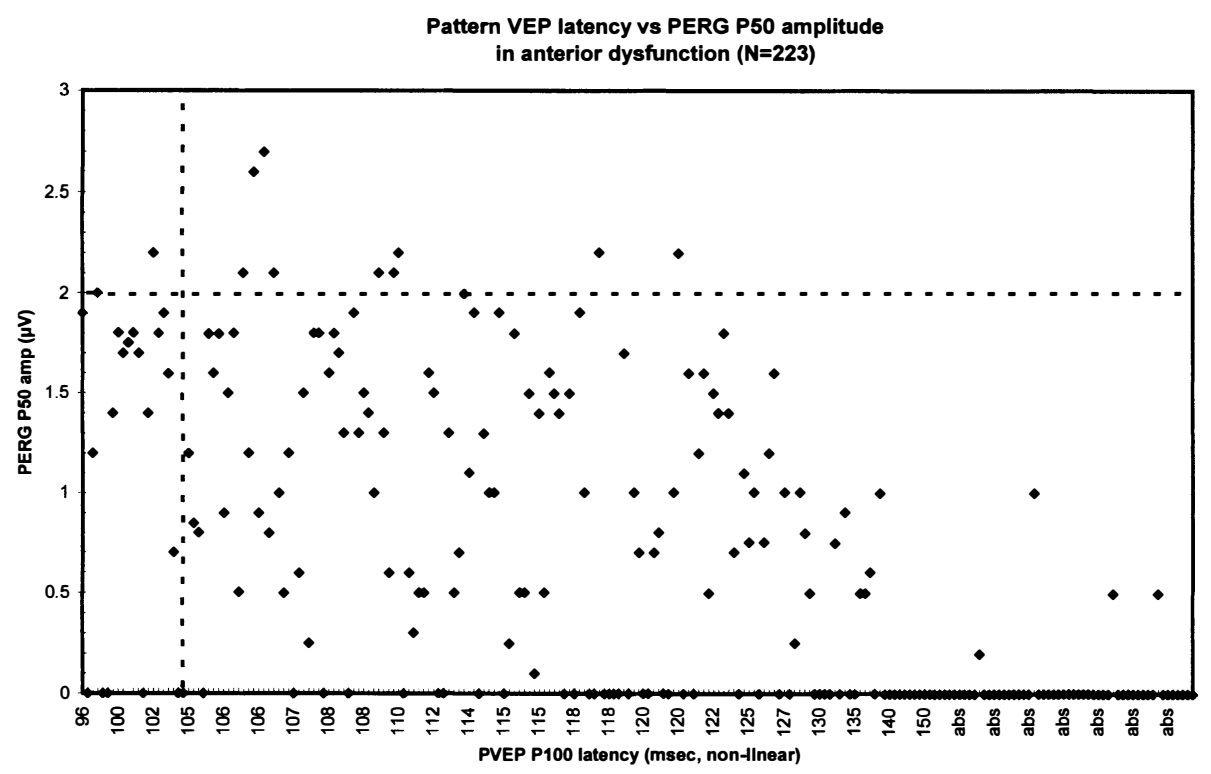

Fig. 1. PERG P50 component amplitudes in 223 eyes with dysfunction anterior to the retinal ganglion cells shown in relation to the latency of the P100 component of the PVEP. The broken horizontal line represents the lower limit of normal P50 amplitude; the broken vertical line the upper limit of normal P100 latency. See text for further details.

Note that the P50 component of the PERG is usually subnormal in these eyes, particularly when the PVEP is delayed (PVEP P100 component latency $>105 \mathrm{~ms}$ ); it is clear that there is a tendency towards a lower P50 component amplitude in relation to an increasing PVEP P100 component latency. Many patients have extinguished PERGs, particularly when there is a marked PVEP latency delay. Also note that some patients have an abnormal P50 component amplitude but a normal PVEP P100 component latency; the PERG P50 component is a more sensitive indicator of macular dysfunction than PVEP latency.
The PERG P50 component, or the overall amplitude of the steady-state PERG (which precludes individual component assessment), was the focus of attention in the early reports of PERG changes in macular dysfunction, ${ }^{28-32}$ all authors finding amplitude reduction with extinction in the more severe cases. This view did not change when the focus expanded to include both P50 and N95 $5^{4,19,33}$ - the N95 component usually showed a degree of amplitude reduction similar in proportion to P50. The present data (Fig. 2) confirm this view, but there are a few patients in whom N95 component amplitude is preserved even though P50 is signifi-

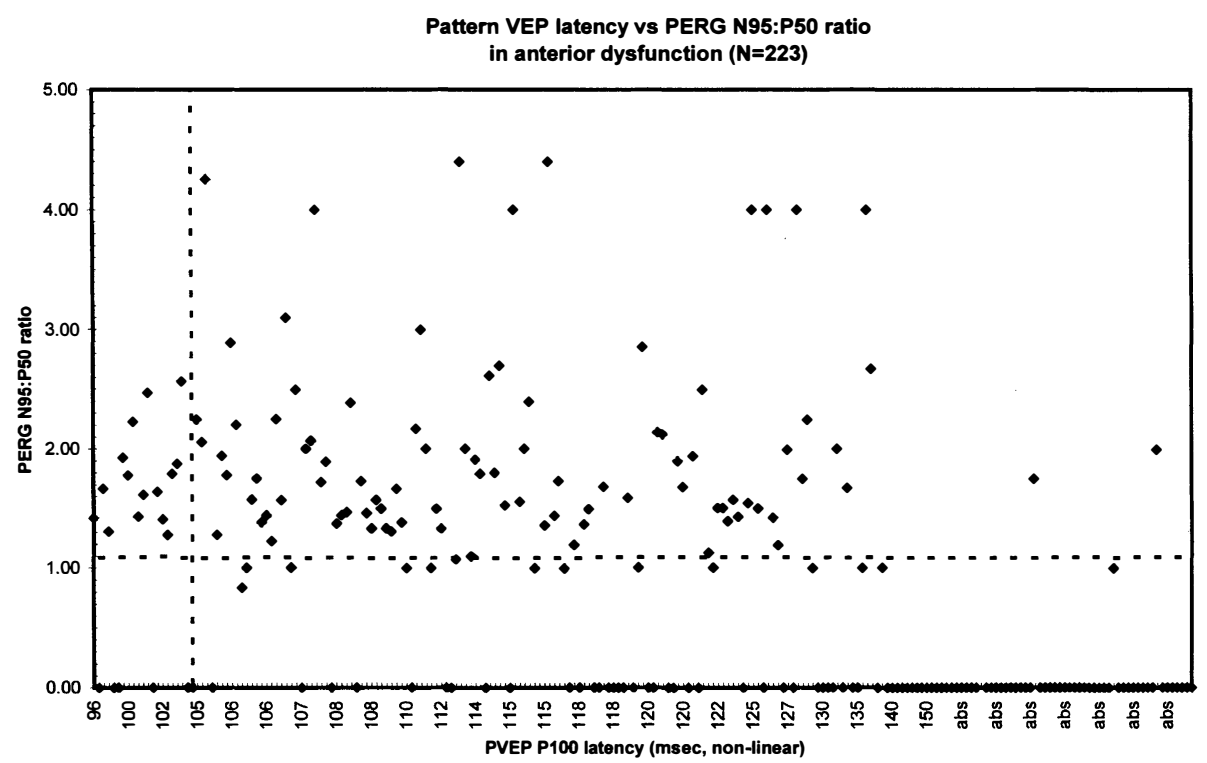

Fig. 2. PERG N95:P50 ratio in 223 eyes with dysfunction anterior to the retinal ganglion cells shown in relation to the latency of the P100 component of the PVEP. The broken horizontal line represents the lower limit of normal N95:P50 ratio, the broken vertical line the upper limit of normal P100 latency. See text for further details. 


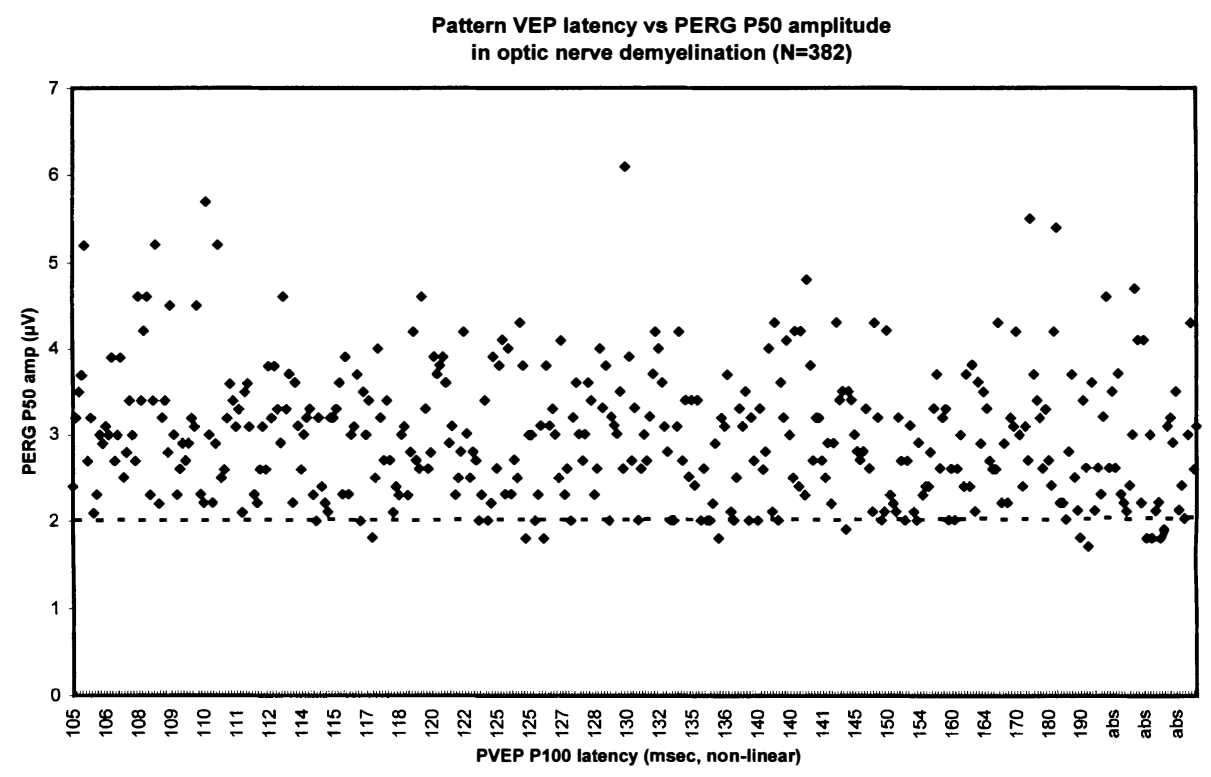

Fig. 3. PERG P50 component amplitudes in 382 eyes with optic nerve demyelination shown in relation to the latency of the P100 component of the PVEP. The broken horizontal line represents the lower limit of normal P50 amplitude; all patients have an abnormal P100 latency as a criterion for optic nerve involvement. See text for further details.

cantly reduced, thereby giving a high N95:P50 ratio. The significance of this is uncertain.

It is of particular importance that when the PVEP is delayed in disease distal (anterior) to the retinal ganglion cells, the P50 component of the PERG is usually subnormal (often unrecordable in severe cases) and there is no trend for the N95:P50 ratio to reduce with increasing P100 component latency, i.e. $\mathrm{N} 95$ is not reduced in isolation from P50. Note the magnitude of PVEP delay present in some patients. Although it has previously been documented that dysfunction of the central retina may give a delayed PVEP, ${ }^{32,34-37}$ this continues to be overlooked in some clinical practice; it is not correct to assume that a delayed PVEP is synonymous with optic nerve disease. In an early series of 67 patients (incorporated in the present data), all 84 eyes with delayed PVEP due to visual pathway dysfunction distal to the optic nerve had abnormalities of the P50 component of the PERG. ${ }^{33}$ Some patients had lens opacities, amblyopia or refractive error as a cause of the PVEP delay. Optic disc swelling of unknown cause precipitated referral in some patients, and in such circumstances examination of ERG, PERG and VEP is recommended. ${ }^{38}$ It should be remembered that papilloedema due to increased intracranial pressure (ICP) does not cause a PVEP abnormality unless secondary optic atrophy has occurred. If an abnormality is present, this reflects the underlying lesion responsible for the rise in ICP. ${ }^{39,40}$

The (full-field) ERG and PERG have a complementary role in diseases of the retina and macula. The PERG enables the direct assessment of central retinal function whereas although the ERG is of great value in the functional assessment of peripheral retinal disease, it is of little diagnostic value in detection of dysfunction confined to the macula. Such a lesion will have a normal ERG ${ }^{31,33,41}$ and the role of the ERG is to assess whether there is significant peripheral retinal involvement, i.e. whether the macular dysfunction is part of a generalised retinopathic process. The PERG also has a role in diseases that principally affect peripheral retina, in that it enables the objective assessment of central retinal function, and may reveal clinically unsuspected evidence of early central retinal dysfunction with possible implications for the prognosis regarding the retention of useful visual acuity. There are no long-term follow-up data at present, but this may be a promising line of future research. The PERG is also of particular interest in neuro-ophthalmic patients; as delayed pattern VEPs commonly occur in retinal/macular dysfunction in addition to the more commonly recognised delays found in optic nerve disease, knowledge of the retinal response to pattern stimulation enables a more accurate interpretation of an abnormal PVEP. It should be remembered that ophthalmoscopy may not necessarily reveal visible evidence of macular dysfunction; for example, in some cases of cone dystrophy the classical bull's eye maculopathy does not occur, and, if the macular changes are mild or absent, the patient may initially have been referred to a neurologist for exclusion of a neurological cause for their visual loss.

The ability of the electrophysiologist to distinguish between optic nerve and macular disease may provide a valuable contribution to the management of the patient. ${ }^{42}$ The objective assessment of macular function provided by the PERG also enables minor ophthalmoscopic changes of uncertain clinical sig- 


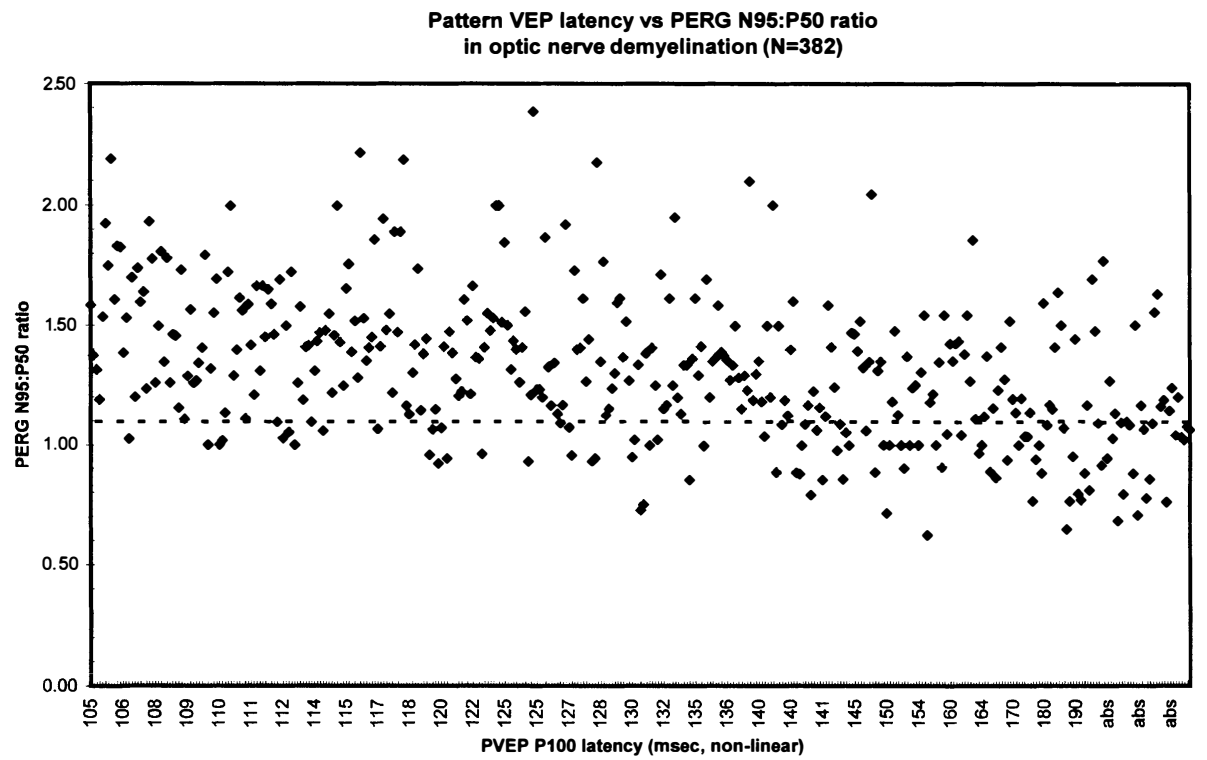

Fig. 4. PERG N95:P50 ratio in 382 eyes with optic nerve demyelination shown in relation to the latency of the P100 component of the PVEP. The broken horizontal line represents the lower limit of normal N95:P50 ratio; all patients have an abnormal P100 latency as a criterion for optic nerve involvement. See text for further details.

nificance to be meaningfully evaluated; this may prevent misdiagnosis in the elderly patient with a non-functioning chromophobe adenoma who presents with visual loss and who has early macular changes on ophthalmoscopy. ${ }^{43}$

\section{Optic Nerve Disease}

The PERG findings in disease of the optic nerve are of great interest. Firstly, optic nerve lesions can be expected eventually to result in retrograde degeneration to the retinal ganglion cells, and if, as has been suggested, the PERG at least partly originates in the retinal ganglion cells, then PERG abnormalities should occur in relation to optic nerve dysfunction. Secondly, from a practical viewpoint in the clinical setting, the PERG is the precursor of the cortical PVEP and gives valuable information regarding the pathways mediating central vision distal to the optic nerve.

The PERG and PVEP findings in a series of patients (382 eyes) with optic nerve demyelination appear in Figs. 3 and 4. Note that all these eyes have an abnormal PVEP latency. Patients with disease of less than 3 weeks' duration have been excluded from this analysis (see below).

The nature of the PERG findings in optic nerve demyelination is completely different from those presented above in dysfunction anterior to the retinal ganglion cells. Most patients, even those with severe delays in the PVEP P100, have a normal P50 component of the PERG (Fig. 3). It is also noteworthy that many patients have a selective reduction confined to the N95 component, as shown by an abnormal N95:P50 ratio (Fig. 4), with a trend to a lower N95:P50 ratio with a greater severity of conduction delay.

The early studies of PERG in optic nerve demyelination confined their attention to the P50 component of the PERG, and reported a variable incidence of abnormality, ${ }^{32,44-47}$ but Porciatti and Von Berger ${ }^{46}$ did note that the negative afterpotential (N95) seemed to be more affected than the main positive component. Some authors concluded that the PERG contributed little significant information. ${ }^{10,48}$ Although absent PERGs were reported in some severely affected eyes, ${ }^{32,49}$ those studies used monocular stimulation, where there are severe problems in controlling fixation in the affected eye, and similar results have not been confirmed by more recent studies using binocular stimulation. Reports of steady-state PERG abnormalities in optic nerve demyelination also appeared. ${ }^{50-52}$

However, when attention was also focused onto the N95 component of the PERG, it became apparent that, if the PERG is abnormal in optic nerve disease, the abnormality may be confined to the N95 component. ${ }^{4,18,19}$ In a previous study of eyes with optic nerve demyelination ${ }^{52}$ only $40 \%$ of PERGs were abnormal, but $85 \%$ of these abnormalities were in relation to the N95 component of the PERG, with no involvement of P50. The findings of that study are confirmed and extended by the current data.

It should also be noted that there may be a marked P50 component abnormality in acute optic neuritis (patients with acute disease are not shown), but this tends to resolve in 2-4 weeks, ${ }^{53,54}$ at which stage there may be a N95 abnormality. As the P50 component of the PERG improves, so the PVEP 


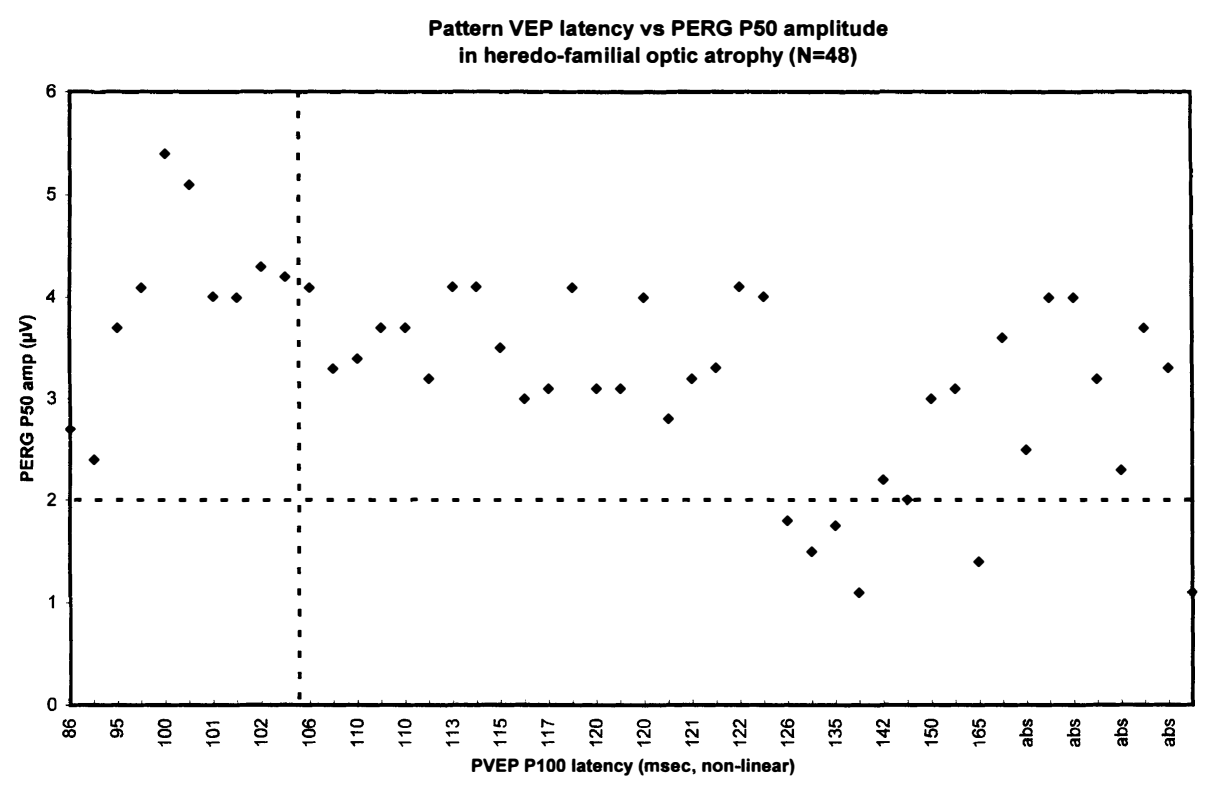

Fig. 5. PERG P50 component amplitudes in 48 eyes with heredo-familial or idiopathic optic atrophy shown in relation to the latency of the P100 component of the PVEP. The broken horizontal line represents the lower limit of normal P50 amplitude; the broken vertical line the upper limit of normal P100 latency. See text for further details.

P100 component latency may increase (deteriorate), suggesting that the process resulting in the temporary PERG P50 component reduction (possible inflammatory change distal to the ganglion cells) is independent of that causing the VEP latency delay (presumed demyelination). In association with this deterioration in VEP latency, there is a recovery of VEP amplitude that may reflect resolution of oedema within the optic nerve.

PERG changes can also occur in other diseases of the optic nerve, but the numbers so far reported are limited. Heredo-familial (or idiopathic) optic atrophy has been reported to show selective loss of the N95 component with normal P50 component in a small number of patients, $6,18,55$ but this has not been confirmed by all authors. ${ }^{56}$ The findings from the author's laboratory (48 eyes) are shown in Figs. 5 and 6. Other causes of optic atrophy have been excluded in these patients.

A few eyes with very severely abnormal PVEPs have involvement of the P50 component of the PERG (Fig. 5), but that the majority of eyes show a marked reduction in N95:P50 ratio (Fig. 6). Other authors have described mean reduction of P50 amplitude in a group of patients including four with juvenile optic atrophy. ${ }^{57} \mathrm{~A}$ previous report of a

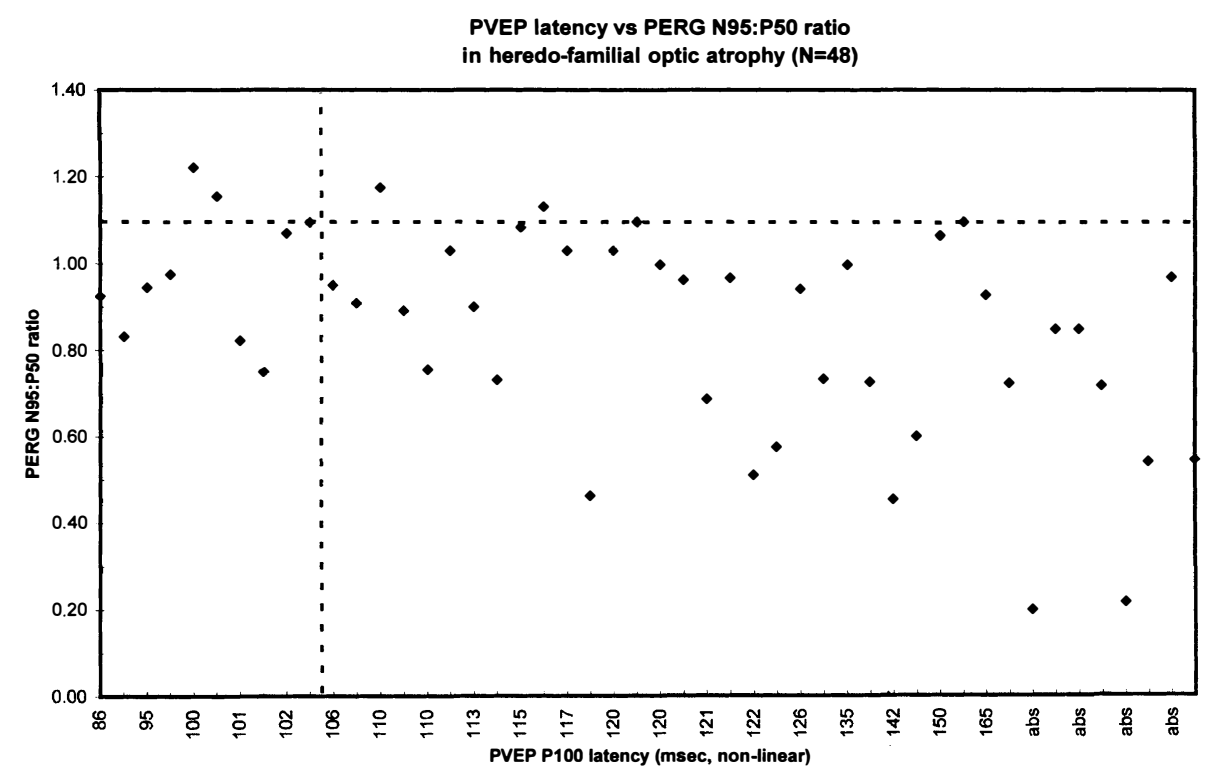

Fig. 6. PERG N95:P50 ratio in 48 eyes with heredo-familial or idiopathic optic atrophy shown in relation to the latency of the P100 component of the PVEP. The broken horizontal line represents the lower limit of normal N95:P50 ratio; the broken vertical line the upper limit of normal P100 latency. See text for further details. 


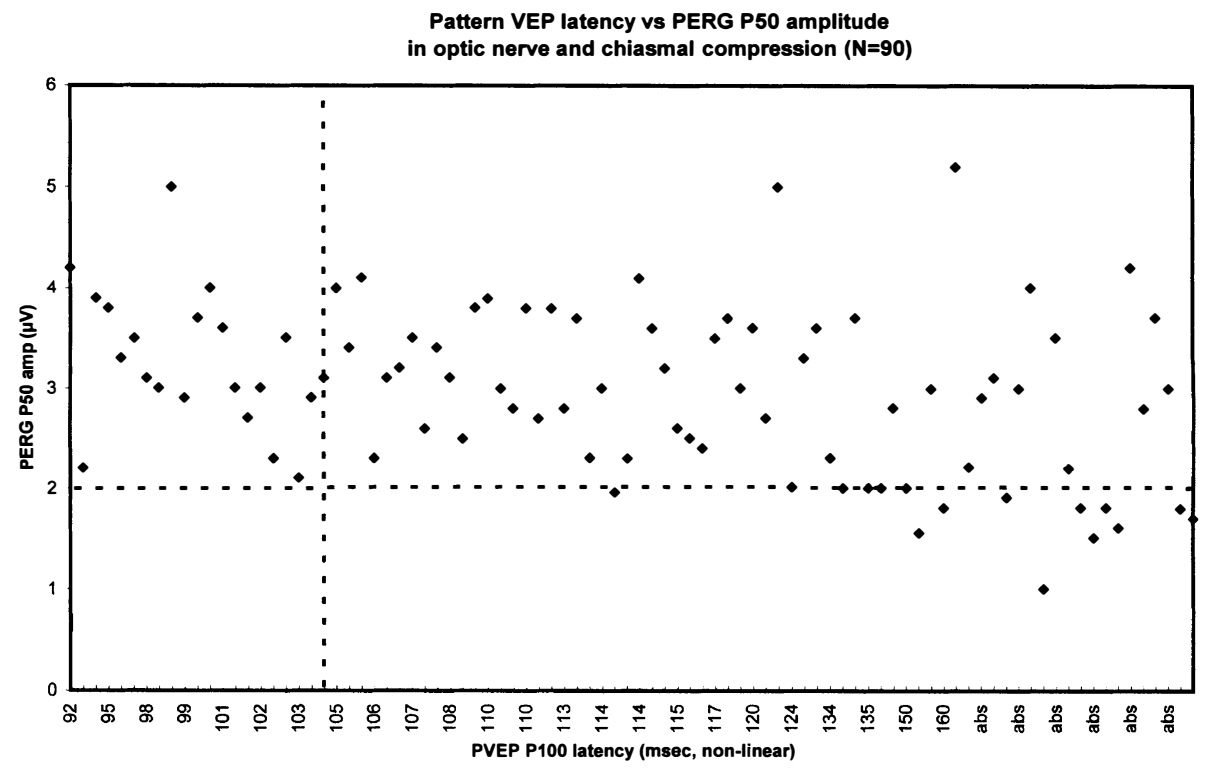

Fig. 7. PERG P50 component amplitudes in 90 eyes with optic nerve compression shown in relation to the latency of the P100 component of the PVEP. The broken horizontal line represents the lower limit of normal P50 amplitude; the broken vertical line the upper limit of normal P100 latency. See text for further details.

patient with Leber's hereditary optic neuropathy, in whom neuroradiological data were also presented, demonstrated prominent bilateral loss of the N95 component with sparing of $\mathrm{P} 50 .{ }^{58}$ Whereas the neuroradiological abnormality was unilateral in relation to the acutely affected eye, the electrophysiological data demonstrated a symmetrical bilateral abnormality; the neuroradiological and electrophysiological data were complementary. The finding of a marked loss of the N95 component in an eye with a history of only a few days suggested primary ganglion cell dysfinction as there had been insufficient time for retrograde degeneration to occur. There have been no longitudinal data published to date in heredo-familial optic atrophy that would enable any possible PERG change in relation to disease duration to be assessed.

Ischaemic optic neuropathy (ION) has been reported to show PERG abnormalities, but there may be a greater degree of P50 involvement than with other optic neuropathies. ${ }^{18}$ A report of the PERG in drusen of the optic nerve head has also appeared that found abnormalities of the N95 component but not of P50. ${ }^{59}$

Compressive optic neve lesions may also give PERG abnormalities. The findings from the author's

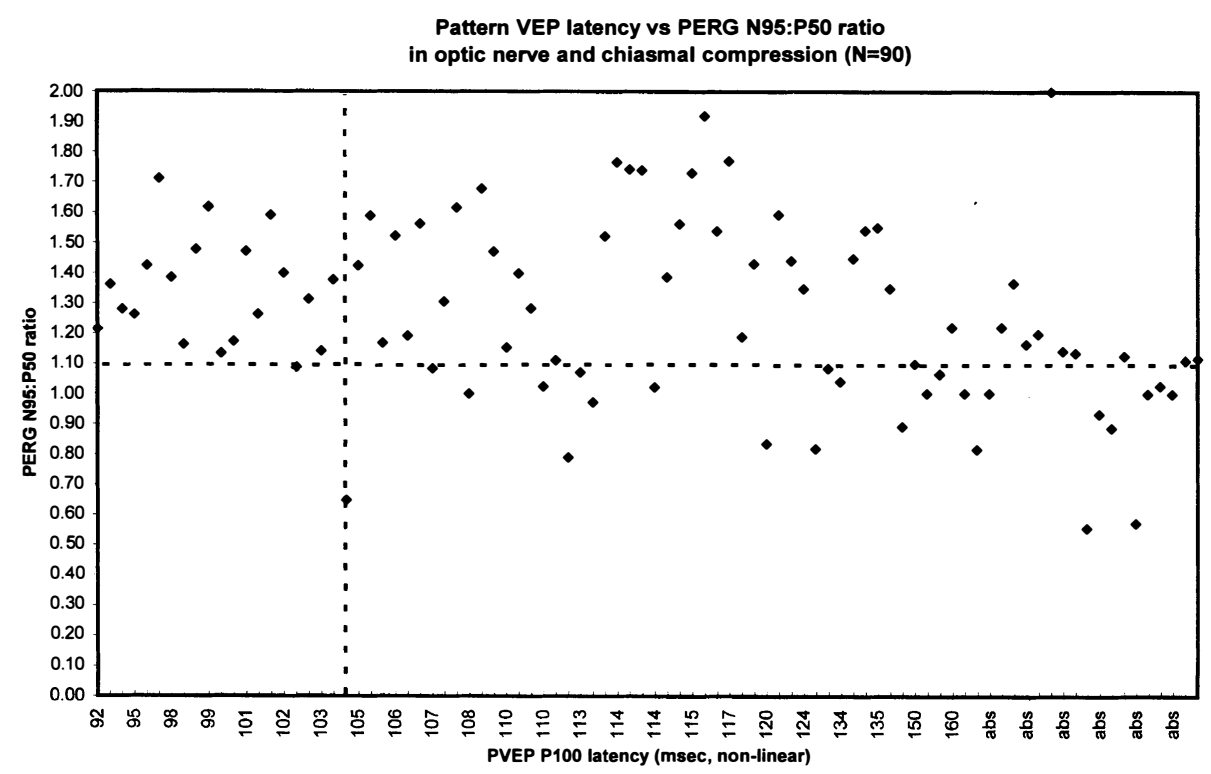

Fig. 8. PERG N95:P50 ratio in 90 eyes with optic nerve compression shown in relation to the latency of the P100 component of the PVEP. The broken horizontal line represents the lower limit of normal N95:P50 ratio; the broken vertical line the upper limit of normal P100 latency. See text for further details. 
Table I. Summary of PERG findings in macular and optic nerve disease

\begin{tabular}{lll}
\hline & Macular disease & Optic nerve disease \\
\hline Abnormality & Yes & Sometimes $(\sim 60 \%$ normal) \\
Component affected & P50 & N95 \\
P50 component latency & May be increased & Occasionally shortened (severe disease). Not increased \\
P50 component amplitude & Reduced (may be extinguished) & $\begin{array}{l}\text { Occasionally reduced (severe disease, and associated with a } \\
\text { greater N95 loss). Not extinguished } \\
\text { May be reduced }\end{array}$ \\
N95:P50 ratio & Normal & May \\
\hline
\end{tabular}

laboratory in patients with optic nerve compression from pituitary tumour or craniopharyngioma ( 90 eyes) are shown in Figs. 7 and 8.

Patients with optic nerve compression rarely show involvement of the P50 component of the PERG unless there is a severe PVEP abnormality, perhaps with extinction of the P100 component (Fig. 7). N95 component abnormalities are more frequent, as shown by a reduced N95:P50 ratio (Fig. 8). One of the patients with an absent PVEP had a large longstanding tumour with a visual acuity of no light perception in one eye (the single P50 amplitude point with a value in the region of $1.0 \mu \mathrm{V}$ ), but there is still a measurable PERG. It is not this author's experience that extinction of the PERG can occur in optic nerve disease, even when very severe, but extinction is not infrequently seen in patients with macular dysfunction (Fig. 1).

It has previously been suggested that the PERG may be a useful prognostic indicator for final visual outcome in the pre-operative assessment of optic nerve compression. ${ }^{60}$ Results from the author's laboratory (Holder, unpublished observations) confirm the usefulness of the PERG, an abnormal PERG recorded as part of pre-operative assessment presumably demonstrating significant retrograde degeneration to the retinal ganglion cells and suggesting that there will be a lack of significant post-operative improvement.

\section{SUMMARY OF CLINICAL FINDINGS}

As is evident from Figs. 3-8, the magnitude of the PVEP P100 latency delay in optic nerve disease frequently exceeds $15 \mathrm{~ms}$, but the PERG P50 component is rarely abnormal. It is therefore of particular clinical relevance to compare these observations with those in patients with delays of similar nature resulting from dysfunction distal to the optic nerve (Figs. 1, 2), where the P50 component is usually abnormal. It should be noted that the specificity of these findings may depend on the use of a high-contrast stimulus. ${ }^{61}$ Although latency changes may occasionally occur with optic nerve disease, P50 component latency does not increase but there may be a shortening of P50 component latency. This is associated with marked N95 component loss. These latency observations again contrast with those in anterior dysfunction where P50 component delays can occur, particularly in patients with macular oedema or serous detachment at the macula. ${ }^{33}$ The findings are summarised in Table I.

The difference in the nature of the PERG abnormality enables the distinction to be made between abnormal PVEPs in optic nerve disease and those in more distal anterior visual pathway dysfunction. An abnormal PVEP with a normal PERG (or an abnormality confined to N95) places the lesion in the optic nerve/ganglion cells. An abnormal PVEP with a moderate or severe P50

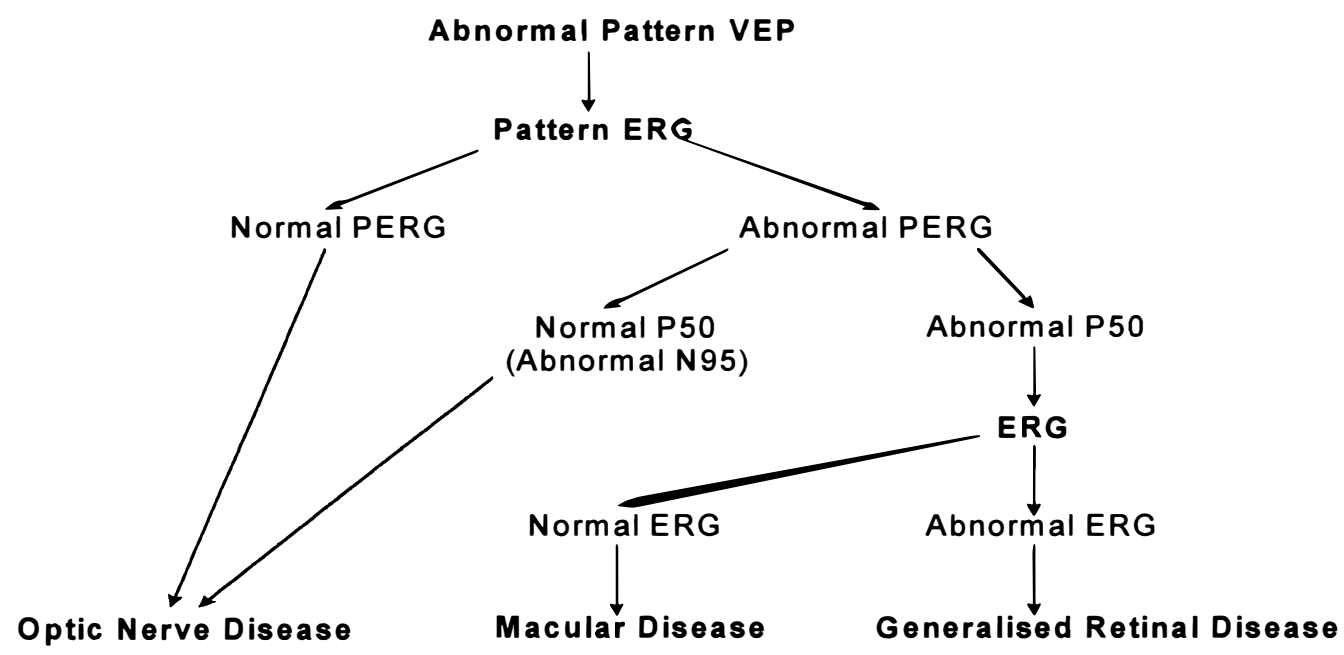

Fig. 9. Test strategy. 
component PERG abnormality suggests more distal dysfunction. These findings led to the development of the diagnostic strategy presented in Fig. 9. It is again noted that these results may apply only when a high-contrast, high-luminance checkerboard stimulus is used, and this type of stimulus is recommended for routine clinical practice.

If there is an abnormal PVEP, and either a normal PERG or a PERG in which the abnormality is confined to N95, then optic nerve disease is suggested. If the P50 component of the PERG is abnormal a full-field ERG is indicated. If this is abnormal there is involvement of the macula as part of generalised retinal dysfunction, but if the ERG is normal dysfunction confined to the macula is implied (assuming that the media are clear and the patient is optimally refracted).

The implications of a misdiagnosis of optic nerve disease cannot be overstressed. The conditions, in the author's experience, that are most likely to be mistaken for optic neuritis are central serous retinopathy and posterior scleritis. In both of these a PERG P50 component abnormality will establish the macula as the site of the dysfunction. It should be remembered that even lack of adequate spectacle correction in a patient referred for PVEP investigation of suspected multiple sclerosis can lead to a PVEP delay, and an abnormal PERG P50 will confirm that the dysfunction is anterior to the optic nerve. It was also demonstrated that the delayed PVEP that can occur in patients with myxoedema, originally thought to reflect optic nerve dysfunction, is accompanied by a delayed, reduced PERG, and that both investigations normalise when the patient becomes euthyroid after treatment with thyroxine. ${ }^{62}$ This suggested that the delayed PVEP in hypothyroidism is probably secondary to reversible central retinal dysfunction.

\section{CONCLUDING REMARKS}

Much of the data presented were obtained with the Arden gold foil electrode, which, in this author's experience, remains the electrode of choice for PERG recording in adults. The ability of the PERG to help distinguish between optic nerve disease and dysfunction distal to the retinal ganglion cells, and to give an objective assessment of macular function, has significantly increased the value of electrophysiological testing both in the objective assessment of those patients with central visual loss and in those patients with ophthalmoscopic changes at the macula of uncertain significance. PERG recording is recommended in any patient with visual symptoms in whom the PVEP is abnormal, as knowledge of the PERG facilitates a more accurate interpretation of the findings.

\section{REFERENCES}

1. Riggs LA, Johnson LP, Schick AML. Electrical responses of the human eye to moving stimulus pattern. Science 1964;144:567-8.

2. Arden GB, Carter RM, Hogg CR, Siegel IM, Margolis S. A gold foil electrode: extending the horizons for clinical electroretinography. Invest Ophthalmol Vis Sci 1979;18:421-6.

3. Dawson WW, Trick GL, Litzkow CA. Improved electrode for electroretinography. Invest Ophthalmol Vis Sci 1979;18:988-91.

4. Holder GE. Significance of abnormal pattern electroretinography in anterior visual pathway dysfunction. $\mathrm{Br}$ J Ophthalmol 1987;71:166-71.

5. Marmor MF, Holder GE, Porciatti V, Trick G, Zrenner E. Guidelines for pattern electroretinography. Recommendations of the International Society for Clinical Electrophysiology of Vision. Doc Ophthalmol 1996;91:291-8.

6. Berninger TA, Arden GB. The pattern electroretinogram. Eye 1988;2(Suppl):S257-83.

7. Maffei L, Fiorentini A. Electroretinographic responses to alternating gratings before and after section of the optic nerve. Science 1981;211:953-5.

8. Maffei L, Fiorentini A, Bisti S, Hollander H. Pattern ERG in the monkey after section of the optic nerve. Exp Brain Res 1985;59:423-5.

9. Trick GL, Wintermeyer DH. Spatial and temporal frequency tuning of pattern reversal retinal potentials. Invest Ophthalmol Vis Sci 1982;23:774-9.

10. Kirkham TH, Coupland SG. The pattern electroretinogram in optic nerve demyelination. Can J Neurol Sci 1983;10:256-60.

11. Riemslag FCC, Ringo JL, Spekreijse H, Verduyn Lunel $\mathrm{H}$. The luminance origin of the pattern electroretinogram in man. J Physiol (Lond) 1985;363:191-209.

12. Berninger TA, Schuurmans RP. Spatial tuning of the pattern ERG across temporal frequency. Doc Ophthalmol 1985;61:17-25.

13. Schuurmans RP, Berninger TA. Luminance and contrast responses recorded in man and cat. Doc Ophthalmol 1985;59:187-97.

14. Van den Berg TJTP, Boltjes B, Spekreijse H. Pattern electroretinogram can be more than the sum of local luminance changes. Doc Ophthalmol 1988;69:307-14.

15. Drasdo N, Thompson DA, Arden GB. A comparison of pattern ERG amplitudes and nuclear layer thickness in different zones of the retina. Clin Vis Sci 1990;5:415-20.

16. Thompson DA, Drasdo N. Computation of the luminance and pattern components of the bar pattern electroretinogram. Doc Ophthalmol 1987;66:233-44.

17. Holder GE. Pattern. ERG abnormalities in anterior visual pathway disease. Electroencephalogr Clin Neurophysiol 1985;61:S135.

18. Holder GE. Abnormalities of the pattern ERG in optic nerve lesions: changes specific for proximal retinal dysfunction. In: Barber C, Blum T, editors. Evoked potentials III. London: Butterworth, 1987:221-4.

19. Ryan S, Arden GB. Electrophysiological discrimination between retinal and optic nerve disorders. Doc Ophthalmol 1988;68:247-55.

20. Tobimatsu S, Celesia G, Cone SB, Gujrati M. Electroretinograms to checkerboard pattern reversal in cats: physiological characteristics and effect of retrograde degeneration of ganglion cells. Electroencephalogr Clin Neurophysiol 1989;73:341-52. 
21. Arden GB, Hogg CR, Holder GE. Gold foil electrodes: a two centre study of electrode reliability. Doc Ophthalmol 1994:86:275-84.

22. Holder GE. Recording the pattern electroretinogram with the Arden gold foil electrode. J Electrophysiol Technol 1988:14:183-90.

23. Thompson DA. Drasdo N. An improved method for using the DTL fibre in electroretinography. Ophthalmic Physiol Opt 1987:7:315-9.

24. Holopigian K. Snow J. Seiple W. Siegel I. Variability of the pattern electroretinogram. Doc Ophthalmol 1988:70:103-15.

25. Prager TC. Fea AM, Sponsel WE, et al. The gold foil electrode in pattern electroretinography. Doc Ophthalmol 1994:86:267-74.

26. Odom JV, Holder GE. Fenghali JG, Cavender S. Pattern electroretinogram intrasession reliability: a two center comparison. Clin Vis Sci 1992:7:263-82.

27. Holder GE. The effects of chiasmal compression on the pattern visual evoked potential. Electrocephalogr Clin Neurophysiol 1978:45:278-80.

28. Sokol S. An electrodiagnostic index of macular degeneration: use of a checkerboard pattern stimulus. Arch Ophthalmol 1972:88:619-24.

29. Groneberg A. Simultaneously recorded retinal and cortical potentials elicited by checkerboard stimuli. Doc Ophthalmol Proc Ser 1980:23:255-62.

30. Sherman J. Simultaneous pattern reversal electroretinograms and visual evoked potentials in diseases of the macula and optic nerve. Ann N Y Acad Sci 1982:388:214-26.

31. Arden GB. Carter RM. MacFarlan A. Pattern and Ganzfeld electroretinograms in macular disease. $\mathrm{Br} \mathrm{J}$ Ophthalmol 1984:68:878-84.

32. Celesia GG. Kaufman D. Pattern ERGs and visual evoked potentials in maculopathies and optic nerve diseases. Invest Ophthalmol Vis Sci 1985:26:726-35.

33. Holder GE. Pattern electroretinography in patients with delayed pattern visual evoked potentials due to distal anterior visual pathway dysfunction. J Neurol Neurosurg Psychiatry 1989:52:1364-8.

34. Lennerstrand G. Delayed visual evoked cortical potentials in retinal disease. Acta Ophthalmol (Copenh) 1982:60:497-504.

35. Holder GE. Chesterton JR. The visual evoked potential in Harada's disease. Neuroophthalmology 1984:4:43-5.

36. Papakostopoulos D. Dean Hart C. Cooper R. Natsikos $\mathrm{V}$. Combined electrophysiological assessment of the visual system in central serous retinopathy. Electroencephalogr Clin Neurophysiol 1984:59:77-80.

37. Bass SJ. Sherman J, Bodis-Wollner I. Nath S. Visual evoked potentials in macular disease. Invest Ophthalmol Vis Sci 1985:26:1071-4.

38. Spalton DJ. Murdoch I. Holder GE. Coxsackie B5 papillitis. J Neurol Neurosurg Psychiatry 1989:52: 1310-1.

39. Asselman P. Chadwick DW, Marsden CD. Visual evoked responses in the diagnosis and management of patients suspected of multiple sclerosis. Brain 1975:98:261-82.

40. Holder GE. Pattern visual evoked potential in patients with posteriorly situated space-occupying lesions. Doc Ophthalmol 1985:59:121-8.

41. Lawwill $T$. The bar pattern electroretinogram for clinical evaluation of the central retina. Am J Ophthalmol 1974:78:224-8.
42. Corbett MC, Shilling JS, Holder GE. The assessment of clinical investigations: the Greenwich Grading System and its application to electrodiagnostic testing in ophthalmology. Eye 1995;9(Suppl):59-64.

43. Holder GE, Bullock PR. Visual evoked potentials in the assessment of patients with non-functioning chromophobe adenomas. J Neurol Neurosurg Psychiatry 1989:52:31-7.

44. Arden GB. Vaegan. Hogg CR. Clinical and experimental evidence that the pattern electroretinogram (PERG) is generated in more proximal retinal layers than the focal electroretinogram (FERG). Ann N Y Acad Sci 1982:388:214-26.

45. Persson HE. Wanger P. Pattern reversal electroretinograms and visual evoked cortical potentials in multiple sclerosis. Br J Ophthalmol 1984:68:760-4.

46. Porciatti V, von Berger GP. Pattern electroretinogram and visual evoked potential in optic nerve disease: early diagnosis and prognosis. Doc Ophthalmol Proc Ser 1984:40:117-26.

47. Serra G. Carreras M. Tugnoli V, Manca M. Cristofori MC. Pattern electroretinogram in multiple sclerosis. J Neurol Neurosurg Psychiatry 1984;47:879-83.

48. Ota I, Miyake Y. The pattern electroretinogram in patients with optic nerve disease. Doc Ophthalmol 1986:62:53-60.

49. Seiple WH. Price MJ. Kupersmith M, Siegel I, Carr R. The pattern electroretinogram in optic nerve disease. Ophthalmology 1983:90:1127-32.

50. Fiorentini A. Maffei L. Pirchio M, Spinelli D, Porciatti $\mathrm{V}$. The ERG in response to alternating gratings in patients with diseases of the peripheral visual pathway. Invest Ophthalmol Vis Sci 1981:21:490-3.

51. Bobak P. Bodis-Wollner I. Harnois C, Maffei L. Mylin L. Podos S. Thornton J. Pattern electroretinograms and visual evoked potentials in glaucoma and multiple sclerosis. Am J Ophthalmol 1983:96:72-83.

52. Holder GE. The incidence of abnormal pattern electroretinography in optic nerve demyelination. Electroencephalogr Clin Neurophysiol 1991:78: $18-26$.

53. Plant GT. Hess RF. Thomas SJ. The pattern evoked electroretinogram in optic neuritis: a combined psychophysical and electrophysiological study. Brain 1986:109:469-90.

54. Berninger TA, Heider W. Pattern electroretinograms in optic neuritis during the acute stage and after remission. Graefes Arch Clin Exp Ophthalmol 1990:228:410-4.

55. Berninger TA. Jaeger W. Krastel H. Electrophysiology and perimetry in autosomal dominant optic atrophy. XXIV ISCEV Meeting. Palermo. 1985.

56. Bach M. Gerling J. Geiger K. Optic atrophy reduces the pattern electroretinogram for both fine and coarse stimulus patterns. Clin Vis Sci 1992:7:327-34.

57. Vaegan. Billson FA. The differential effect of optic nerve disease on pattern and focal electroretinograms. Doc Ophthalmol 1987:65:45-56.

58. Mauguiere F. Holder GE. Luxon LM. Pottinger RC. Abnormal waveforms and diagnostic yield of evoked potentials. In: Osselton JW. Binnie CD. Cooper R. Fowler CJ. Mauguiere F. Prior P. editors. Clinical neurophysiology: EMG. nerve conduction and evoked potentials. Oxford: Butterworth-Heinemann. 1995: 431-81.

59. Scholl GB. Song H-S. Winkler DE. Wray SH. The 
pattern visual evoked potential and pattern electroretinogram in drusen-associated optic neuropathy. Arch Ophthalmol 1992;110:75-81.

60. Kaufmann D, Wray SH, Lorance R, Woods M. An analysis of the pathophysiology and the development of treatment strategies for compressive optic nerve lesions using pattern electroretinogram and visual evoked potential. Neurology 1986;36(Suppl 1):232.
61. Holder GE. Pattern electroretinography in the evaluation of glaucoma and in optic nerve dysfunction. In: Heckenlively JR, Arden GB. Principles and practice of clinical electrophysiology of vision. St Louis: MosbyYear Book, 1991:549-56.

62. Holder GE, Condon JR. Pattern visual evoked potentials and pattern electroretinograms in hypothyroidism. Doc Ophthalmol 1989;73:127-31. 